\title{
Fast and Simple Method for Evaluation of Polarization Correction to Propagation Constant of Arbitrary Order Guided Modes in Optical Fibers with Arbitrary Refractive Index Profile
}

\begin{abstract}
Anton Bourdine
Department of Communication Lines, Povolzhskiy State University of Telecommunications and Informatics (PSUTI), 77 Moscow Avenue, Samara 443090, Russia
\end{abstract}

Correspondence should be addressed to Anton Bourdine; bourdine@yandex.ru

Received 5 May 2015; Accepted 29 June 2015

Academic Editor: Xiao-Qiao He

Copyright (C) 2015 Anton Bourdine. This is an open access article distributed under the Creative Commons Attribution License, which permits unrestricted use, distribution, and reproduction in any medium, provided the original work is properly cited.

\begin{abstract}
This work presents fast and simple method for evaluation of polarization correction to scalar propagation constant of arbitrary order guided modes propagating over weakly guiding optical fibers. Proposed solution is based on earlier on developed modified Gaussian approximation extended for analysis of weakly guiding optical fibers with arbitrary refractive index profile in the core region bounded by single solid outer cladding. Some results are presented that illustrate the decreasing of computational error during the estimation of propagation constant when polarization corrections are taken into account. Analytical expressions for the first and second derivatives of polarization correction are derived and presented.
\end{abstract}

\section{Introduction}

The weakly guiding approximation based on scalar wave equation (Helmholtz equation) is widely used in the most well-known approximate methods and their various modifications for analysis of optical waveguides. Because the difference between refractive indexes of the core and the cladding of conventional telecommunication silica optical fibers is less than $1 \%$, they are weakly guiding. Therefore this approximation providing a passage from the vector wave equation to simplified scalar wave equation is applied as basic assumption in various approximate methods for computing of optical fiber mode parameters under sufficient accuracy. However these solutions are not exact, because they do not take into account polarization effects, and there are some applications requiring more high accuracy. The problem can be solved by adding polarization correction $\delta \beta$ to scalar propagation constant $\beta$ that would improve accuracy of mode propagation constant calculation. Polarization correction is described in detail by Snyder and Love [1]. It relates to "exact" and "approximate" propagation constants by following simple ratio (Equation (32.22) in [1]):

$$
\beta_{\text {exact }}^{2}=\beta^{2}-\delta \beta
$$

This work presents fast and simple method for evaluation of polarization correction to scalar propagation constant of arbitrary order guided modes propagating over weakly guiding optical fibers. Proposed solution is based on earlier on developed modified Gaussian approximation extended for analysis of weakly guiding optical fibers with arbitrary refractive index profile in the core region bounded by single solid outer cladding. Some results are presented that illustrate the decreasing of computational error during the estimation of propagation constant when polarization corrections are taken into account. Analytical expressions for the first and second derivatives of polarization correction are derived and presented. 


\section{Polarization Correction: General Information}

Generally polarization correction to approximate scalar propagation constant for modes propagating over optical waveguide with arbitrary cross section and refractive index profile can be found by following well-known perturbation theory formula (Equation (32.24) in [1]):

$$
\delta \beta=\frac{a(2 \Delta)^{3 / 2}}{2 V} \frac{\int_{A_{\infty}}\left(\nabla_{t} \cdot \vec{E}\right) \vec{E} \cdot \nabla_{t} f(x, y) d A}{\int_{A_{\infty}}|\vec{E}|^{2} d A},
$$

where $a$ is core radius, $\Delta=\left(n_{\max }^{2}-n_{N}^{2}\right) / 2 n_{\max }^{2}$ is profile height parameter, $n_{\max }$ is maximal value of refractive index in the core region, $n_{N}$ is outer cladding refractive index, $V=\operatorname{kan}_{\max } \sqrt{2 \Delta}$ is normalized frequency, $k=2 \pi / \lambda$ is wavenumber, $\lambda$ is wavelength, and $\vec{E}$ is transverse electric field [1-6]:

$$
\vec{E}=F_{m}^{(l)}(r)\left[\left\{\begin{array}{l}
\cos (l \varphi) \\
\sin (l \varphi)
\end{array}\right\} \widehat{x} \mp\left\{\begin{array}{l}
\sin (l \varphi) \\
\cos (l \varphi)
\end{array}\right\} \widehat{y}\right],
$$

where $F_{m}^{(l)}(r)$ is radial mode field distribution, $l$ is azimuthal mode number $(l=0,1,2, \ldots), m$ is radial mode number $(m=$ $1,2,3, \ldots), \widehat{x}$ and $\hat{y}$ are unit vectors in the directions of the $x$ and $y$-axis, $A_{\infty}$ is infinite cross section square, and $\nabla_{t}$ is the transverse component of nabla differential operator:

$$
\nabla_{t}=\widehat{x} \frac{\partial}{\partial x}+\widehat{y} \frac{\partial}{\partial y}
$$

$f(r)$ is profile function related with refractive index profile $n(R)$ by well-known expression [1-5]:

$$
n^{2}(R)=n_{0}^{2}[1-2 \Delta \cdot f(R)] .
$$

Passage to optical fibers will split expression (2) into following integrals (Table (14.1) in [1]):

$$
\begin{aligned}
& I_{1}=\frac{(2 \Delta)^{3 / 2}}{4 a V} \frac{\int_{0}^{\infty} F_{m}^{(l)}\left(\partial F_{m}^{(l)} / \partial R\right)(\partial f / \partial R) R d R}{\int_{0}^{\infty}\left[F_{m}^{(l)}\right]^{2} R d R} \\
& I_{2}=\frac{l(2 \Delta)^{3 / 2}}{4 a V} \frac{\int_{0}^{\infty}\left[F_{m}^{(l)}\right]^{2}(\partial f / \partial R) d R}{\int_{0}^{\infty}\left[F_{m}^{(l)}\right]^{2} R d R}
\end{aligned}
$$

where $R=r / a$ is normalized radius. Here polarization correction to scalar propagation constant of particular order guided mode in optical fiber is represented in the form of mentioned two integrals combination. Table 1 compares the relations between LP modes and conventional modes and contains corresponding polarization corrections defined by (6a) and (6b) integrals (Table (14.1) in [1]).

By substituting radial mode field distribution $F_{m}^{(l)}$ and profile function $f(R)$ into integrals (6a) and (6b) the final expression for polarization correction can be obtained. For example, below there is polarization correction $\delta \beta$ to the
TABLE 1

\begin{tabular}{lcc}
\hline LP mode & Conventional mode & $\delta \beta$ \\
\hline $\mathrm{LP}_{0 m}(l=0)$ & $\mathrm{HE}_{1 m}(l=0)$ & $I_{1}$ \\
\hline \multirow{2}{*}{$\mathrm{LP}_{1 m}(l=1)$} & $\mathrm{TE}_{0 m}$ & 0 \\
& $\mathrm{TM}_{0 m}$ & $2\left(I_{1}+I_{2}\right)$ \\
& $\mathrm{HE}_{2 m}$ & $I_{1}-I_{2}$ \\
& $\mathrm{EH}_{2 m}$ & $I_{1}+I_{2}$ \\
\hline $\mathrm{LP}_{l m}(l>1)$ & $\mathrm{HE}_{l+1, m}$ & $I_{1}-I_{2}$ \\
& $\mathrm{EH}_{l+1, m}$ & $I_{1}+I_{2}$ \\
\hline
\end{tabular}

mode $\mathrm{HE}_{1 m}\left(\mathrm{LP}_{0 m}\right)$ propagation constant for weakly guiding step index optical fiber (Table (14.3) in [1]):

$$
\delta \beta=-\frac{(2 \Delta)^{3 / 2}}{2 a} \frac{U^{2} W}{V^{2}} \frac{K_{0}(W)}{K_{1}(W)},
$$

where $U$ and $W$ are core and cladding mode parameters: $U^{2}+$ $W^{2}=V^{2} ; K_{l}$ is second kind modified Bessel function.

And polarization correction to the fundamental mode propagation constant for the graded optical fiber with ideal infinite parabolic index profile is described by following expression (Table (14.2) in [1]):

$$
\delta \beta=-\frac{(2 \Delta)^{3 / 2}}{2 a V} .
$$

Both ideal step and ideal parabolic refractive index profiles correspond to exact solutions of scalar wave equation that completely describe mode field distribution. However unlike ideal profiles, analysis of real optical fibers requires a passage to more complicated refractive index profiles differing from ideal forms by local defects and refractive index fluctuations [3,7]. Also a passage to higher order guided modes should be required in the case of a few or multimode optical fibers.

In this work, the mentioned problem is solved by use of earlier on developed extension of modified Gaussian approximation (EMGA). EMGA provides computing of transmission parameters of arbitrary order guided modes. It was developed for analysis of weakly guiding silica optical fibers with axialsymmetric arbitrary refractive index profile in the core region bounded by one solid outer cladding.

\section{Extension of Modified Gaussian Approximation}

EMGA is based on conventional well-known Gaussian approximation [1] of radial mode field distribution $F_{m}^{(l)}(R)$ in the weakly guiding optical waveguide with an arbitrary refractive index profile by the well-known Laguerre-Gauss function expression [1-6], describing a mode field distribution in weakly guiding optical waveguide with ideal infinite parabolic index profile:

$$
F_{m}^{(l)}(R)=\left(\frac{R}{R_{0}}\right)^{l} L_{m-1}^{(l)}\left(\frac{R^{2}}{R_{0}^{2}}\right) \exp \left(\frac{-R^{2}}{2 R_{0}^{2}}\right),
$$


where $L_{m-1}^{(l)}$ is Laguerre polynomial, $R_{0}=\rho_{0} / a$ is equivalent normalized mode field radius, and $\rho_{0}$ is equivalent mode field radius.

EMGA leads to equivalent normalized mode field radius $R_{0}$ estimation by solving a characteristic equation, which is derived from propagation constant variational expression under following passage to square core mode parameter $U^{2}$ variational expression, written for analyzed weakly guiding optical waveguide with given refractive index profile. $R_{0}$ is variational parameter. It is basic for Gaussian approximation and completely defines mode transmission parameters.

The most well-known methods utilized variational approach with conventional Gaussian approximation and their various modifications $[1,8-14]$ are based on representation of optical fiber refractive index profile by simple power functions, while the real commercial fiber profiles have more complex form with local defects and refractive index fluctuations [3, 7]. Some methods solve this problem by using representation of profile or/and mode fields by series expansions together with adding other variational parameters [1-15]. This leads to increasing the number of equations in characteristic set or number of series terms for complex profiles and higher order modes. That is the main reason why most methods for analysis of optical fibers are concerned with computing of transmission parameters only for the fundamental mode. Even in the case of generalization of conventional Gaussian approximation proposed by Snyder and Love (Section 15.6 and Table (15.4) in [1]) analytical variational expressions were obtained only for modes $\mathrm{LP}_{l 1}$ with the radial order $m=1$ due to elimination of Laguerre polynomial $L_{m-1}^{(l)}$.

Unlike the abovementioned known methods, in EMGA the stratification method approach [3] is applied for representation of complicated profile form corresponding to real commercial telecommunication optical fibers. Here optical fiber with an arbitrary axial-symmetric refractive index profile is considered as multicladding optical fiber. Therefore, refractive index profile inside core region can be represented in the form of the set of $N$ layers in which the refractive index stays a constant:

$$
n(R)= \begin{cases}n_{k}, & R_{k}=\frac{k}{N}, 0 \leq k \leq N-1 \\ n_{N}, & 1<R \leq+\infty,\end{cases}
$$

and any profile function $f(R)$ can be written in terms of profile parameter $h_{k}$ :

$$
f(R)= \begin{cases}h_{k}, & R_{k}=\frac{k}{N}, 0 \leq k \leq N-1 \\ 1, & 1<R \leq+\infty,\end{cases}
$$

where

$$
h_{k}=\frac{n_{\max }^{2}-n_{k}^{2}}{n_{\max }^{2}-n_{N}^{2}}
$$

where $n_{k}$ is refractive index of $k$ layer $(k=0, \ldots, N), n_{\max }$ is the maximal core refractive index, and $n_{N}$ is cladding refractive index.
This approach based on stratification method makes EMGA to be versatile that provides ability of analysis of real commercial optical fibers with nonideal disturbed refractive index profile.

Another problem occurring during a passage from wellknown integral variational expressions for propagation constant or core mode parameter (Equations (15.4) and (15.18) in [1]) to the analytical formulas for higher order modes is a presence of nonstandard integrals with product of Laguerre polynomials of nonequal degree and order in the numerator. Here it is solved by representation of Laguerre polynomial in the form of finite power series according to its definition [16].

Proposed approach permits writing the variational expression for core mode parameter $U$ and characteristic equation for normalized equivalent mode field radius $\partial U^{2} / \partial R_{0}=0$ in the form of finite nested sums for any order mode $\mathrm{LP}_{l m}$ as follows:

$$
\begin{aligned}
& U^{2}=\frac{(m-1) !}{(l+m-1) !}\left\{\frac{Q}{R_{0}^{2}}\right. \\
&\left.+V^{2}\left[X_{0}+\sum_{k=0}^{N-1} h_{k}\left(X_{1}-X_{2}\right)\right]\right\} ; \\
& X_{0}=\exp \left(-\frac{1}{R_{0}^{2}}\right) \sum_{q=0}^{2 m-2} D_{q} \sum_{p=0}^{l+q} \frac{(l+q) !}{p ! R_{0}^{2 p}} ; \\
& X_{1}=\exp \left(-\frac{k^{2}}{N^{2} R_{0}^{2}}\right) \sum_{q=0}^{2 m-2} D_{q} \sum_{p=0}^{l+q} \frac{(l+q) !}{p ! R_{0}^{2 p}} \frac{k^{2 p}}{N^{2 p}} ; \\
& X_{2}=\exp \left(-\frac{(k+1)^{2}}{N^{2} R_{0}^{2}}\right)^{2 m-2} \sum_{q=0}^{l+2} D_{q} \sum_{p=0}^{l+q} \frac{(l+q) !}{p ! R_{0}^{2 p}} \frac{(k+1)^{2 p}}{N^{2 p}} ; \\
&-+V^{2}\left[S_{0}+\sum_{k=0}^{N-1} h_{k}\left(S_{1}-S_{2}\right)\right]=0 ; \\
& S_{0}=\exp \left(-\frac{1}{R_{0}^{2}}\right) \sum_{q=0}^{2 m-2} D_{q} \sum_{p=0}^{l+q} \frac{(l+q) !}{p ! R_{0}^{2 p}}\left(1-p R_{0}^{2}\right) ; \\
&+2 l^{2} \sum_{q=0}^{2 m-2} D_{q}(q+l-1) !-4 l \sum_{q=0}^{2 m-2} C_{q}(q+l) ! \\
& S_{1}=\exp \left(-\frac{k^{2}}{N^{2} R_{0}^{2}}\right) \sum_{q=0}^{2 m-2} D_{q} \\
& \frac{(k+1)^{2 p}}{N^{2 p}}\left(\frac{(k+1)^{2}}{N^{2}}-p R_{0}^{2}\right) ; \\
& \cdot \sum_{p=0}^{l+q} \frac{(l+q) !}{p ! R_{0}^{2 p}} \frac{k^{2 p}}{N^{2 p}}\left(\frac{k^{2}}{N^{2}}-p R_{0}^{2}\right) ; \\
& S_{2}=\exp \left(-\frac{(k+1)^{2}}{N^{2} R_{0}^{2}}\right) \sum_{q=0}^{2 m-2} D_{q} \sum_{p=0}^{l+q} \frac{(l+q) !}{p ! R_{0}^{2 p}}
\end{aligned}
$$


where

$$
\begin{aligned}
& D_{q}=\sum_{p=\max (0, q-m+1)}^{\min (q, m-1)} b_{p}^{(l, m-1)} b_{q-p}^{(l, m-1)} ; \\
& C_{q}=\sum_{p=\max (0, q-m+1)}^{\min (q, m-1)} b_{p}^{(l, m-1)} b_{q-p}^{(l+1, m-1)} ;
\end{aligned}
$$

and $b_{p}^{(l, m)}$ is coefficient of polynomial representation in the form of power series $[15,16]$ :

$$
\begin{aligned}
L_{m}^{(l)}(x) & =\sum_{q=0}^{m} b_{q}^{(l, m)} x^{q} ; \\
b_{q}^{(l, m)} & =(-1)^{q} \frac{(l+m) !}{(l+q) !(m-q) ! q !} .
\end{aligned}
$$

Therefore analysis of weakly guiding single-cladding optical fiber with an arbitrary profile leads to the following. Refractive index profile is represented by profile function (10) in the form of $N$ layers. Fiber parameters and mode orders $l$ and $m$ are substituted to characteristic equation (14). By means of numerical solution (14), the normalized equivalent mode field radius $R_{0}$ will be obtained. Then $R_{0}$ is substituted into expression (12), and mode core parameter $U$ is estimated, which permits evaluating the propagation constant $\beta$ for guided mode $\mathrm{LP}_{l m}$ by the well-known expression [1-6]:

$$
\beta^{2}=k_{0}^{2} n_{\max }^{2}-\frac{U^{2}}{a^{2}} .
$$

Solution of characteristic equation (14) is correct under normalized frequency $V>1$, and it should satisfy the guided mode cutoff condition [1-6]:

$$
k_{0} n_{N}<\beta \leq k_{0} n_{\max } .
$$

Optical confinement factor $P_{\text {core }}$ can be considered as the second criterion for identification of the ghost solutions:

$$
P_{\text {co }}^{(l m)} \geq 0.5 \text {. }
$$

By taking into account Gaussian approximation parameter $P_{\text {core }}$ is defined by analytical expression derived from the generalized integral form for weakly guiding optical fibers presented in [1]:

$$
P_{\mathrm{co}}^{(l m)}=\frac{(m-1) !}{(l+m-1) !} \sum_{q=0}^{2 m-2} D_{q}(l+q) ! \cdot W_{q},
$$

where

$$
W_{q}=1-\exp \left(-\frac{1}{R_{0}^{2}}\right) \sum_{p=0}^{l+q} \frac{1}{p ! R_{0}^{2 p}} .
$$

Therefore EMGA provides computation of transmission parameters of arbitrary order guided modes propagating over weakly guiding optical fibers with arbitrary axial-symmetric refractive index profile under high accuracy demonstrated in [17] also due to taking into account researched fiber profile local distortions.

\section{Polarization Correction to the Propagation Constant of Guided Modes in Weakly Guiding Optical Fiber with an Arbitrary Refractive Index Profile}

According to the abovementioned, it is supposed that the optical fiber is analyzed by EMGA based on Gaussian approximation. Therefore substitution of (9) into integral expressions (6a) and (6b) will lead the denominator integral to the simple ratio of azimuthal and radial mode numbers $l$ and $m[16]$ :

$$
\begin{aligned}
\int_{0}^{\infty} & \left(\frac{R}{R_{0}}\right)^{2 l}\left[L_{m-1}^{(l)}\left(\frac{R^{2}}{R_{0}^{2}}\right)\right]^{2} \exp \left(\frac{-R^{2}}{R_{0}^{2}}\right) R d R \\
& =\frac{(l+m-1) !}{(m-1) !} .
\end{aligned}
$$

By applying Laguerre polynomial recurrence and derivation formulas [16] and carrying out awkward algebraic transformations, the analytical expression for radial mode field distribution derivation $\partial F_{m}^{(l)}(R) / \partial R$ in the numerator of integral (6a) can be written in the form

$$
\begin{aligned}
& \frac{d F_{m}^{(l)}}{d R}=\frac{R^{l-1}}{R_{0}^{l}} \exp \left(-\frac{R^{2}}{2 R_{0}^{2}}\right) \\
& \cdot\left[\left(\frac{R^{2}}{R_{0}^{2}}+l\right) L_{m-1}^{(l)}\left(\frac{R^{2}}{R_{0}^{2}}\right)-\frac{2 R^{2}}{R_{0}^{2}} L_{m-1}^{(l+1)}\left(\frac{R^{2}}{R_{0}^{2}}\right)\right] \\
& =\left(\frac{l}{R}+\frac{R}{R_{0}^{2}}\right) F_{m}^{(l)}-\frac{2}{R_{0}} F_{m}^{(l+1)} .
\end{aligned}
$$

According to the abovementioned, EMGA uses stratification method approach for refractive index profile representation inside the fiber core region by finite number of $N$ layers. As a result profile function $f(R)$ is defined by expression (10). By taking into account the great number of layers $(N>$ 100 [7]) required for detailed description of real optical fiber refractive index profile, the derivative of profile function from the numerator integral of expression (6a) can be written in the following form:

$$
\begin{aligned}
& \left.\frac{\partial f}{\partial R}\right|_{R=R_{k}}=\frac{f\left(R_{k+1}\right)-f\left(R_{k}\right)}{R_{k+1}-R_{k}} \\
& \quad= \begin{cases}N\left(h_{k+1}-h_{k-1}\right), & R_{k}=\frac{k}{N}, 0 \leq k \leq N-1 \\
0, & 1<R_{k} \leq+\infty .\end{cases}
\end{aligned}
$$

As a result the numerator integral of (6a) is led to finite sum, and expression (6a) by taking into account (23), (24), and (25) will be rewritten as follows:

$$
\begin{aligned}
I_{1} & =\frac{(m-1) !}{(l+m-1) !} \frac{(2 \Delta)^{3 / 2}}{4 a V} \\
& \left.\cdot N \sum_{k=0}^{N-1}\left(h_{k+1}-h_{k}\right)\left[F_{m}^{(l)} R_{k} \frac{\partial F_{m}^{(l)}}{\partial R}\right]\right|_{R_{k}=k / N} .
\end{aligned}
$$




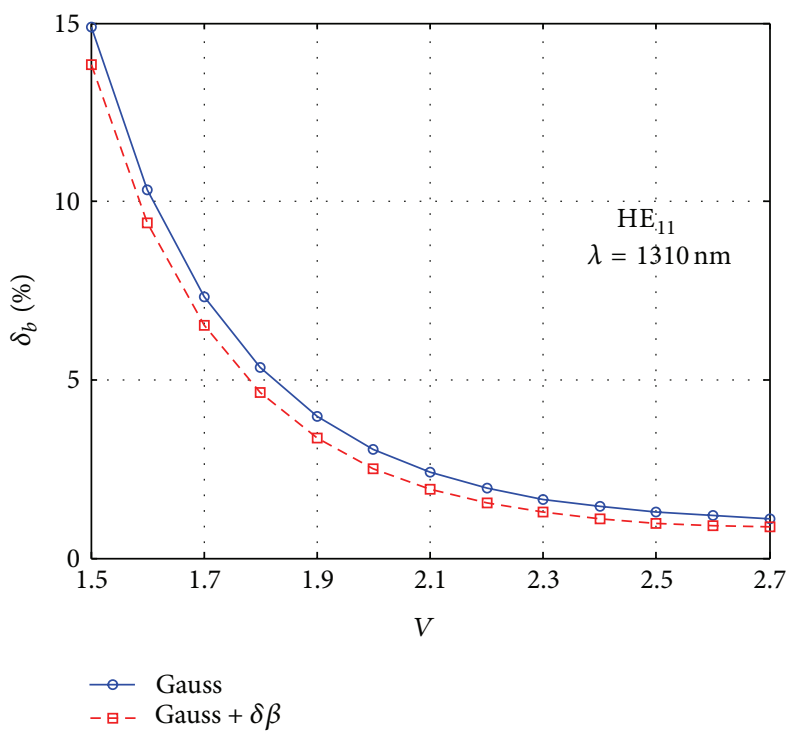

(a)

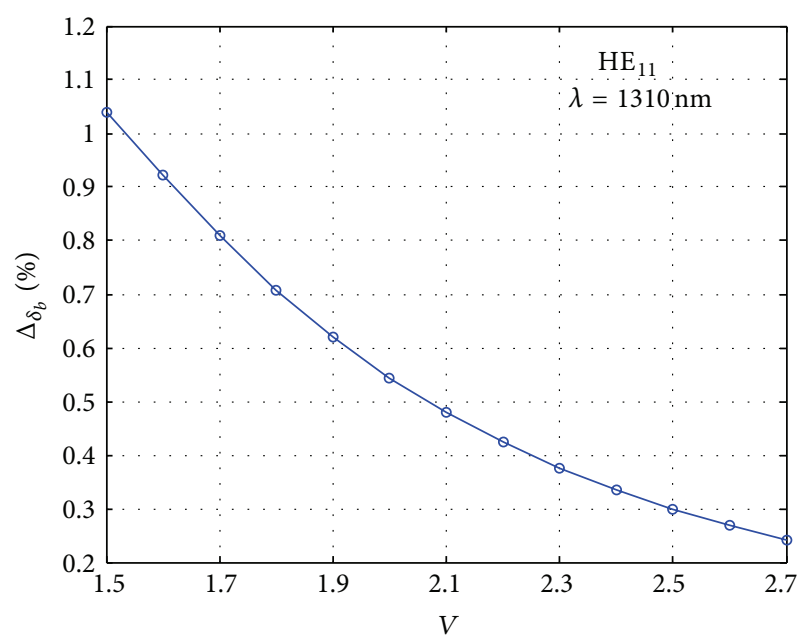

(b)

FIGURE 1: Error reducing of calculation of the fundamental mode $\mathrm{HE}_{11}$ normalized propagation constant produced by EMGA by polarization correction under low value of normalized frequency: (a) relative error, with and without polarization correction; (b) difference of relative error curves.

After substituting (24) into (26) the final analytic expression for integral $I_{1}$ will be obtained:

$$
\begin{gathered}
I_{1}=\frac{(m-1) !}{(l+m-1) !} \frac{N(2 \Delta)^{3 / 2}}{2 a V R_{0}^{2}} \sum_{k=0}^{N-1}\left(h_{k+1}-h_{k}\right)\left\{\left[F_{m}^{(l)}\right]^{2}\right. \\
\left.\cdot\left[l+\frac{R_{k}^{2}}{R_{0}^{2}}-\frac{2 R_{k}^{2}}{R_{0}^{2}} \cdot \frac{L_{m-1}^{(l+1)}\left(R_{k}^{2} / R_{0}^{2}\right)}{L_{m-1}^{(l)}\left(R_{k}^{2} / R_{0}^{2}\right)}\right]\right\}\left.\right|_{R_{k}=k / N} .
\end{gathered}
$$

By applying the same approach to $I_{2}$, after substituting (23) and (24) into (6b) the expression for integral $I_{2}$ will be written in the following analytical form:

$$
\begin{aligned}
I_{2} & =\frac{l(m-1) !}{(l+m-1) !} \frac{(2 \Delta)^{3 / 2}}{4 a V} \\
& \left.\cdot N \sum_{k=0}^{N-1}\left(h_{k+1}-h_{k}\right)\left\{\left[F_{m}^{(l)}\right]^{2}\right\}\right|_{R_{k}=k / N} .
\end{aligned}
$$

\section{Results and Discussion}

The "worst" case from the point of view of EMGA application for analysis of optical fibers, ideal step index refractive index profile, will be considered for estimation of polarization correction influence on error reducing. According to $[3,4,18]$, it corresponds to computing of fundamental mode propagation constant $\beta$ under the low values of normalized frequency near $V=2.405$. Therefore conventional silica step index optical fiber with pure silica cladding and 3.1\% Germanium doped core was considered. Normalized frequency range $V=$ $1.5 \cdots 2.7$ was researched under wavelength $\lambda=1310 \mathrm{~nm}$.
Some results of error comparison between exact solution of wave equation and approximate values of normalized propagation constant obtained for the fundamental mode $\mathrm{HE}_{11}$ by EMGA without and by taking into account polarization correction are presented in Figure 1. Here polarization correction provides error reducing on $0.25 \cdots 1.05 \%$ over researched low value normalized frequency range. The influence of polarization correction on the error reducing decreases under normalized frequency enhancement.

Further results of the higher order mode propagation constant estimation produced by exact solution and EMGA without and with polarization correction near the particular value of normalized frequency corresponding to mode cutoff condition were also compared. Normalized propagation constant relative error curves $\delta_{b}(V)$ obtained for guided mode $\mathrm{HE}_{21}$ and their difference are presented in Figure 2. Here polarization correction provides reducing of relative error on $0.5 \cdots 1.5 \%$ under the researched range of $V=$ $3 \cdots 5$. Following increasing value of normalized frequency also reduces influence of polarization correction: it decreases relative error not more than $0.1 \cdots 0.2 \%$.

Following passage to higher order guided modes also demonstrates decreasing of polarization correction influence on propagation constant error reducing. Differences of relative error curves for guided higher order modes $\mathrm{HE}_{31}$ and $\mathrm{HE}_{41}$ are shown in Figure 3. Here it is less than $0.2 \%$ for mode $\mathrm{HE}_{31}$ and $0.004 \%$ for mode $\mathrm{HE}_{41}$ even under values of $V$ near the cutoff condition corresponding to the mentioned guided modes.

Further the same analysis was produced for step index multimode optical fiber 50/125 also with pure silica cladding and $3.1 \%$ Germanium doped core over wavelength range $\lambda=800 \cdots 1350 \mathrm{~nm}$. Relative error curves calculated for the 


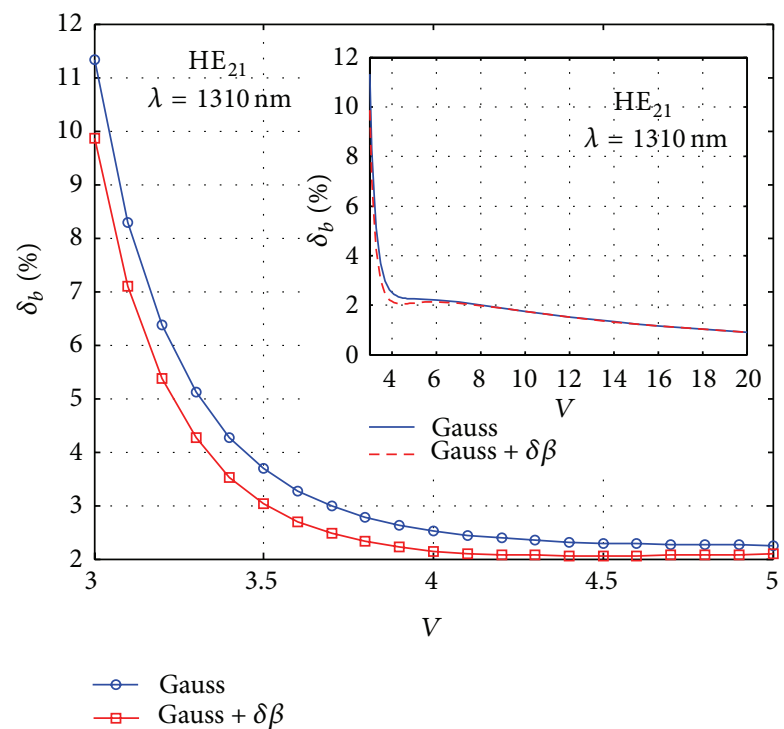

(a)

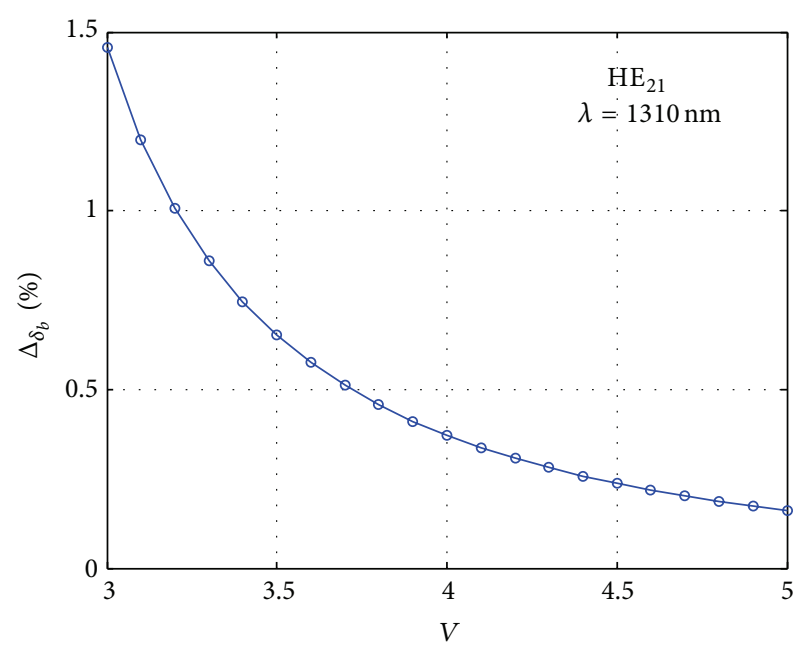

(b)

FIGURE 2: Error reducing of calculation of the higher order mode $\mathrm{HE}_{21}$ normalized propagation constant produced by EMGA by polarization correction under low value of normalized frequency: (a) relative error, with and without polarization correction; (b) difference of relative error curves.

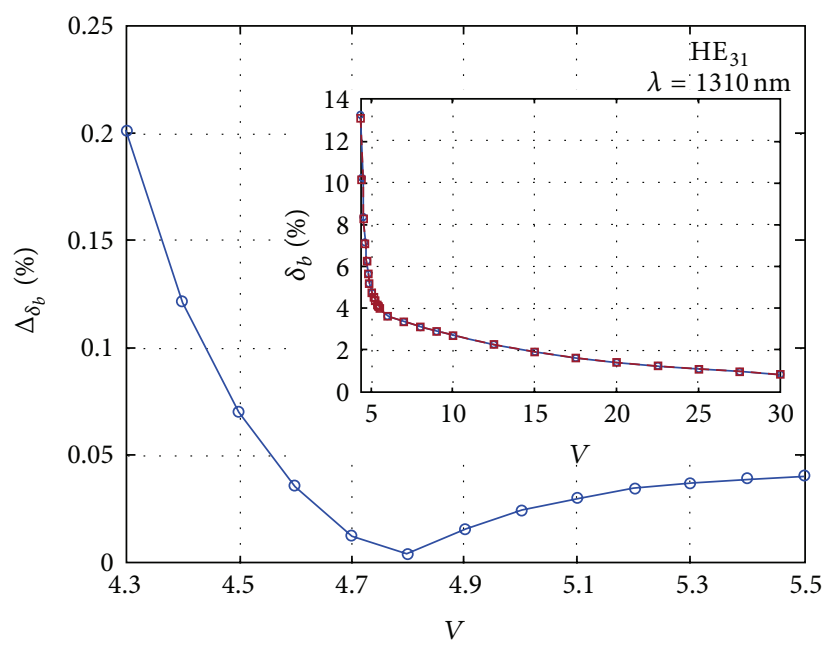

(a)

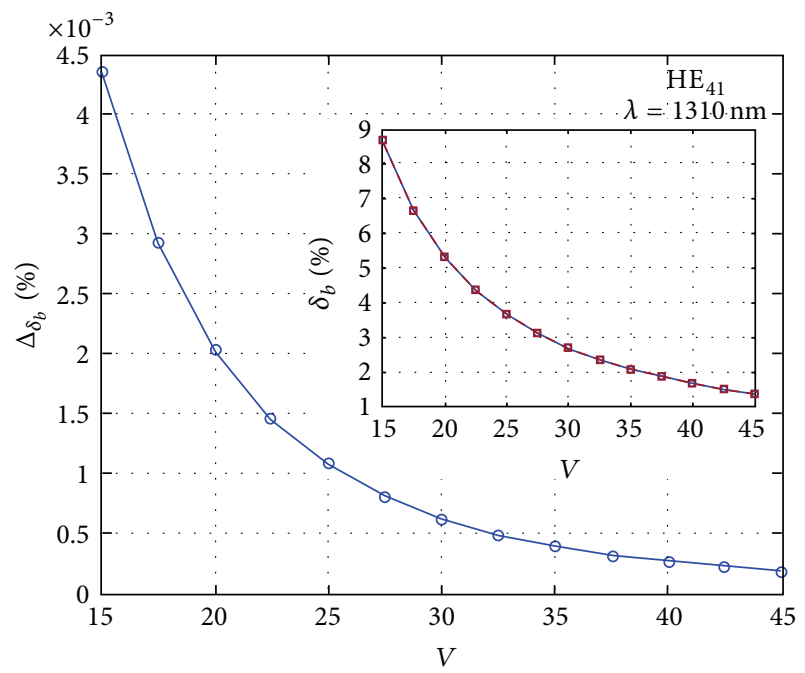

(b)

FIGURE 3: Difference of relative error curves of calculation of the higher order modes normalized propagation constant produced by EMGA with and without polarization correction: (a) $\mathrm{HE}_{31}$ mode; (b) $\mathrm{HE}_{41}$ mode.

fundamental mode $\mathrm{HE}_{11}$ and their difference are represented in Figure 4. Here normalized frequency $V$ reaches high values $13.5 \cdots 23$ over researched wavelength range. That is why the error is so small and is less than $0.32 \%$, and polarization correction ensures its decrease to only $0.0005 \cdots 0.00035 \%$, while for higher order modes it helps to improve accuracy up to $0.01 \cdots 0.1 \%$ depending on mode order. For example, differences of relative error curves for guided higher order modes $\mathrm{HE}_{23}$ and $\mathrm{HE}_{32}$ are shown in Figure 5. For mode $\mathrm{HE}_{23}$ the error reduces by $0.02 \cdots 0.12 \%$ during wavelength growing and corresponding normalized frequency $V$ decreasing under fixed core diameter value, while for mode $\mathrm{HE}_{32}$ it reaches $0.005 \cdots 0.04 \%$.

According to obtained results, the main influence of polarization correction $\delta \beta$ on reducing of normalized propagation constant calculation error corresponds to lower order guided modes under critical values of normalized frequency $V$ near cutoff condition (low core diameter or wavelength 


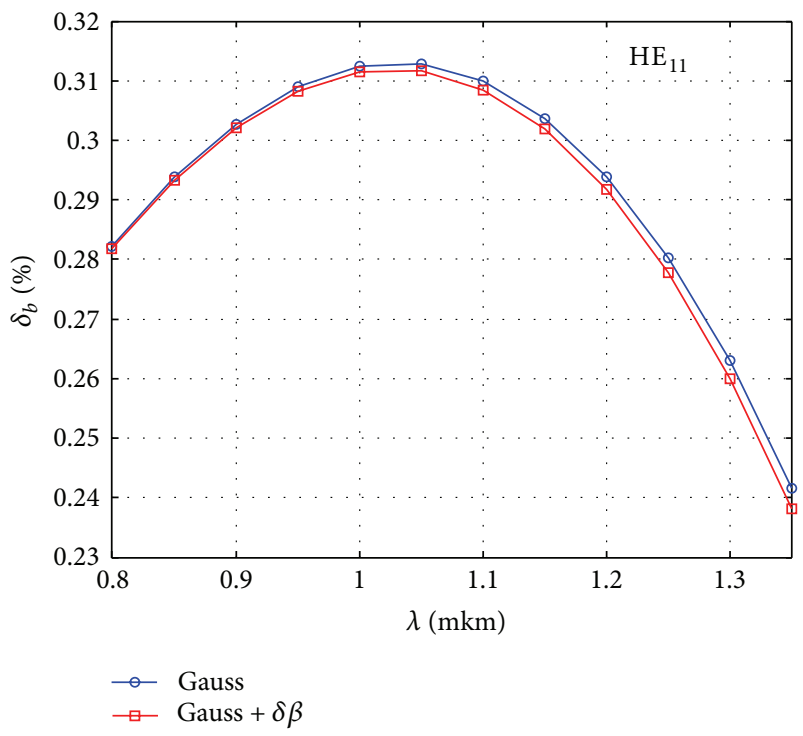

(a)

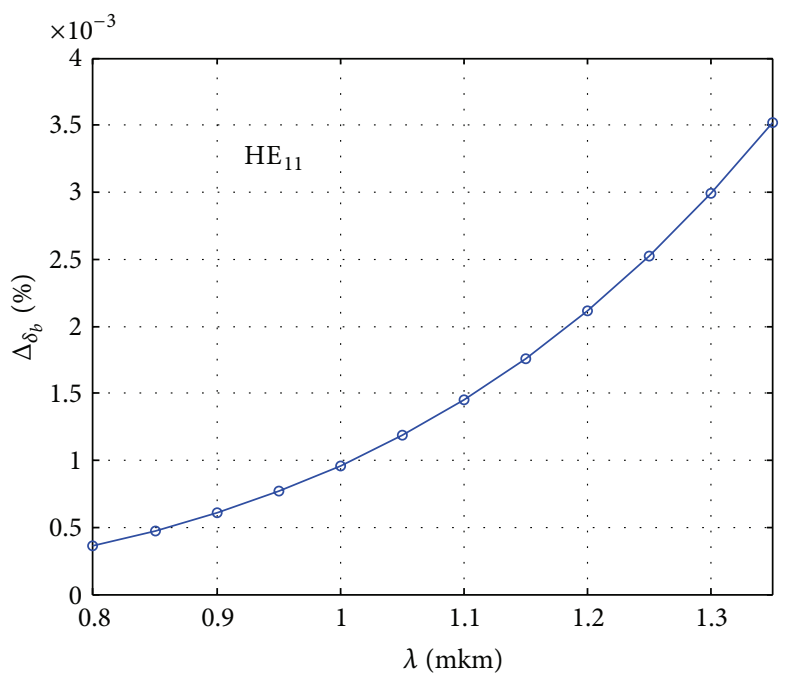

(b)

FIGURE 4: Error reducing of calculation of the fundamental mode $\mathrm{HE}_{11}$ normalized propagation constant produced by EMGA by polarization correction: (a) relative error, with and without polarization correction; (b) difference of relative error curves.

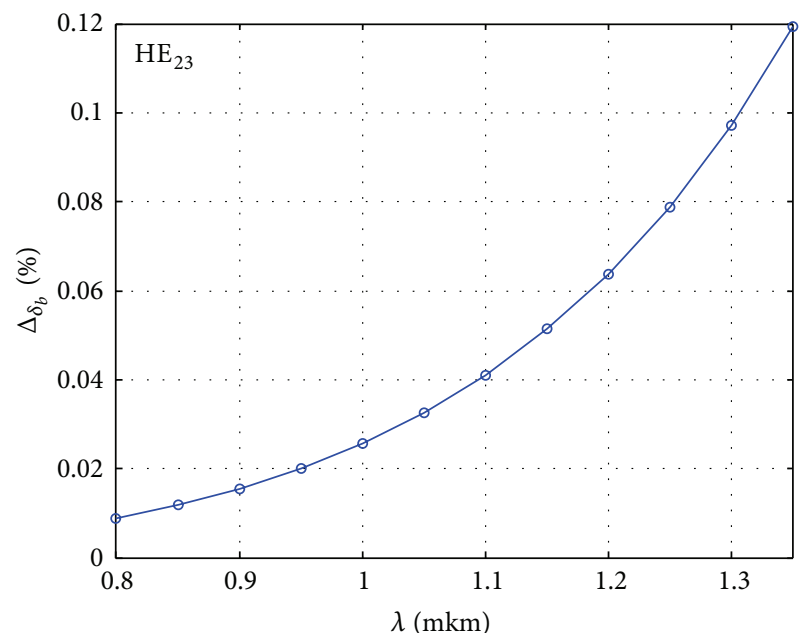

(a)

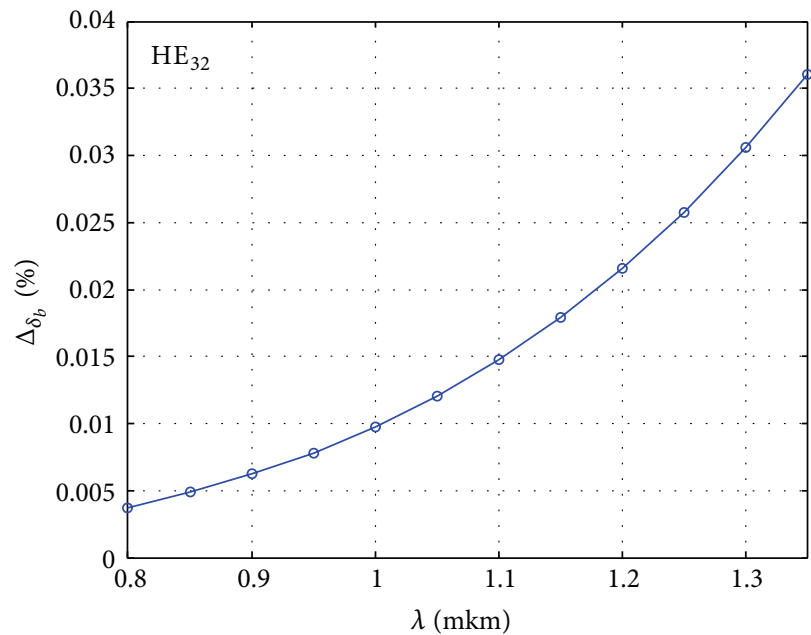

(b)

FIGURE 5: Difference of relative error curves of calculation of the higher order modes normalized propagation constant produced by EMGA with and without polarization correction: (a) $\mathrm{HE}_{23}$ mode; (b) $\mathrm{HE}_{32}$ mode.

near mode cutoff wavelength). Under described conditions, relative error can be reduced by up to $1 \%$. By taking into account the following passage to the first and second propagation constant derivatives $\partial \beta / \partial \lambda$ and $\partial^{2} \beta / \partial \lambda^{2}$ required for estimation of mode dispersion parameters, it would much reduce the errors of computing of mode delay and chromatic dispersion of guided modes with particular order.

Derived analytical expressions for polarization correction to the scalar propagation constant estimated by EMGA have analytical form and do not require high-performance computers. For example, computing time of any order guided mode "exact" propagation constant $\beta_{\text {exact }}$ by taking into account polarization correction $\delta \beta$ at the particular wavelength $\lambda$ and the number of layers for refractive index profile representation in the fiber core region $N>300$ is less than $1 \mathrm{~s}$ under the following computing resource parameters: AMD Phenom II x4 965, 3.4 HHz, RAM 4 Gb, Microsoft Windows 7.0 Professional, and Matlab 6.1.

Derived analytical formulas for polarization correction $\delta \beta$ and its derivatives represented in Appendix provide 
both improving of EMGA accuracy and ability of taking into account polarization effects and specifically polarization mode dispersion that is especially topical for problems of simulation of Multi-Gigabit data transmission over fiber optic links

\section{Conclusion}

Based on EMGA fast and simple method for calculation of polarization correction to any order mode propagation constant in weakly guiding optical fiber with an arbitrary axial-symmetric refractive index profile is introduced. Some results of estimation of propagation constant computing error decreasing by taking into account polarization correction are demonstrated. The main influence of polarization correction $\delta \beta$ on reducing of propagation constant calculation error was noticed for lower order guided modes under critical values of normalized frequency $V$ near cutoff condition (low core diameter or wavelength near mode cutoff wavelength). Derived analytical formulas for polarization correction $\delta \beta$ and its derivatives provide both improving of EMGA accuracy and ability of taking into account polarization effects and specifically polarization mode dispersion that is especially topical for problems of simulation of Multi-Gigabit data transmission over fiber optic links.

\section{Appendix}

\section{First and Second Derivatives of Polarization Correction}

A passage from the propagation constant to mode delay and chromatic dispersion requires expressions for polarization correction first and second derivatives. First, let us rewrite formula (27a) in the compacted following form:

$$
I_{1}=\frac{(m-1) !}{(l+m-1) !} \sum_{k=0}^{N-1} G_{k}^{(1)},
$$

where

$$
\begin{gathered}
G_{k}^{(1)}=P\left(h_{k+1}-h_{k}\right)\left[F_{m-1}^{(l)}\right]^{2} Q_{k} ; \\
P=\frac{N(2 \Delta)^{3 / 2}}{2 a V R_{0}^{2}} ; \\
Q_{k}=\left.\left[l+\frac{R_{k}^{2}}{R_{0}^{2}}-\frac{2 R_{k}^{2}}{R_{0}^{2}} \cdot \frac{L_{m-1}^{(l+1)}\left(R_{k}^{2} / R_{0}^{2}\right)}{L_{m-1}^{(l)}\left(R_{k}^{2} / R_{0}^{2}\right)}\right]\right|_{R_{k}=k / N} .
\end{gathered}
$$

It provides representing the first and second derivatives of the first component of polarization correction $I_{1}$ also in the form of finite sum:

$$
\begin{aligned}
\frac{\partial I_{1}}{\partial \lambda} & =\frac{(m-1) !}{(l+m-1) !} \sum_{k=0}^{N-1} \frac{\partial G_{k}^{(1)}}{\partial \lambda} \\
\frac{\partial^{2} I_{1}}{\partial \lambda^{2}} & =\frac{(m-1) !}{(l+m-1) !} \sum_{k=0}^{N-1} \frac{\partial^{2} G_{k}^{(1)}}{\partial \lambda^{2}} .
\end{aligned}
$$

After differentiation and carrying out necessary transformations the derivatives of parameter $G_{k}^{(1)}$ are led to the following form:

$$
\begin{aligned}
& \frac{\partial G_{k}^{(1)}}{\partial \lambda}=P\left[F_{m}^{(l)}\right]^{2} Q\left(\frac{\partial h_{k+1}}{\partial \lambda}-\frac{\partial h_{k}}{\partial \lambda}\right)+G_{k}^{(1)}\left(\frac{1}{P} \frac{\partial P}{\partial \lambda}\right. \\
& \left.+\frac{2}{F_{m}^{(l)}} \frac{\partial F_{m}^{(l)}}{\partial \lambda}+\frac{1}{Q} \frac{\partial Q}{\partial \lambda}\right) \\
& \frac{\partial^{2} G_{k}^{(1)}}{\partial \lambda^{2}}=P\left[F_{m}^{(l)}\right]^{2} Q\left[\left(\frac{\partial h_{k+1}}{\partial \lambda}-\frac{\partial h_{k}}{\partial \lambda}\right)\right. \\
& +\left(\frac{1}{P} \frac{\partial P}{\partial \lambda}+\frac{2}{F_{m}^{(l)}} \frac{\partial F_{m}^{(l)}}{\partial \lambda}+\frac{1}{Q} \frac{\partial Q}{\partial \lambda}\right)+\frac{\partial^{2} h_{k+1}}{\partial \lambda^{2}} \\
& \left.+\frac{\partial^{2} h_{k}}{\partial \lambda^{2}}\right]+\frac{\partial G_{k}^{(1)}}{\partial \lambda}\left(\frac{1}{P} \frac{\partial P}{\partial \lambda}+\frac{2}{F_{m}^{(l)}} \frac{\partial F_{m}^{(l)}}{\partial \lambda}+\frac{1}{Q} \frac{\partial Q}{\partial \lambda}\right) \\
& +G_{k}^{(1)}\left\{\frac{1}{P}\left[\frac{\partial^{2} P}{\partial \lambda^{2}}-\frac{1}{P}\left(\frac{\partial P}{\partial \lambda}\right)^{2}\right]\right. \\
& \left.+\frac{1}{Q}\left[\frac{\partial^{2} Q}{\partial \lambda^{2}}-\frac{1}{Q}\left(\frac{\partial Q}{\partial \lambda}\right)^{2}\right]\right\} \cdot \\
& F_{m}^{(l)}
\end{aligned}
$$

The derivatives of parameter $P$ are expressed as follows:

$$
\begin{aligned}
\frac{\partial P}{\partial \lambda} & =P\left[\frac{3}{2 \Delta} \frac{\partial \Delta}{\partial \lambda}-\frac{1}{R_{0} V}\left(2 V \frac{\partial R_{0}}{\partial \lambda}+R_{0} \frac{\partial V}{\partial \lambda}\right)\right] ; \\
\frac{\partial^{2} P}{\partial \lambda^{2}} & =\frac{1}{P}\left(\frac{\partial P}{\partial \lambda}\right)^{2}+P\left\{\frac{3}{2 \Delta}\left[\frac{\partial^{2} \Delta}{\partial \lambda^{2}}-\frac{1}{\Delta}\left(\frac{\partial \Delta}{\partial \lambda}\right)^{2}\right]\right. \\
+ & \frac{2}{R_{0}}\left[\frac{1}{R_{0}}\left(\frac{\partial R_{0}}{\partial \lambda}\right)^{2}-\frac{\partial^{2} R_{0}}{\partial \lambda^{2}}\right] \\
+ & \left.\frac{1}{V}\left[\frac{1}{V}\left(\frac{\partial V}{\partial \lambda}\right)^{2}-\frac{\partial^{2} V}{\partial \lambda^{2}}\right]\right\} .
\end{aligned}
$$

First- and second-order derivatives of parameter $Q$ are defined as follows:

$$
\begin{aligned}
& \frac{\partial Q_{k}}{\partial \lambda}=\frac{2 R^{2}}{R_{0}^{2}}\left\{\frac { L _ { m - 1 } ^ { ( l + 1 ) } ( R _ { k } ^ { 2 } / R _ { 0 } ^ { 2 } ) } { L _ { m - 1 } ^ { ( l ) } ( R _ { k } ^ { 2 } / R _ { 0 } ^ { 2 } ) } \left[\frac{2}{R_{0}} \frac{\partial R_{0}}{\partial \lambda}\right.\right. \\
& \left.+\frac{1}{L_{m-1}^{(l)}\left(R_{k}^{2} / R_{0}^{2}\right)} \frac{\partial}{\partial \lambda}\left[L_{m-1}^{(l)}\left(\frac{R_{k}^{2}}{R_{0}^{2}}\right)\right]\right]-\frac{1}{R_{0}} \frac{\partial R_{0}}{\partial \lambda} \\
& \left.-\frac{1}{L_{m-1}^{(l+1)}\left(R_{k}^{2} / R_{0}^{2}\right)} \frac{\partial}{\partial \lambda}\left[L_{m-1}^{(l+1)}\left(\frac{R_{k}^{2}}{R_{0}^{2}}\right)\right]\right\} ;
\end{aligned}
$$




$$
\begin{gathered}
\frac{\partial^{2} Q_{k}}{\partial \lambda^{2}}=-\frac{2}{R_{0}} \frac{\partial R_{0}}{\partial \lambda} \frac{\partial Q}{\partial \lambda}+\frac{2 R_{k}^{2}}{R_{0}^{2}}\left\{\frac { 1 } { R _ { 0 } } \left[\frac{1}{R_{0}}\left(\frac{\partial R_{0}}{\partial \lambda}\right)^{2}\right.\right. \\
\left.-\frac{\partial^{2} R_{0}}{\partial \lambda^{2}}\right]-\frac{1}{L_{m-1}^{(l)}\left(R_{k}^{2} / R_{0}^{2}\right)} \frac{\partial^{2}}{\partial \lambda^{2}}\left[L_{m-1}^{(l+1)}\left(\frac{R_{k}^{2}}{R_{0}^{2}}\right)\right] \\
+\frac{2}{L_{m-1}^{(l)}\left(R_{k}^{2} / R_{0}^{2}\right)}\left[\frac{1}{R_{0}} \frac{\partial R_{0}}{\partial \lambda}+\frac{1}{L_{m-1}^{(l)}\left(R_{k}^{2} / R_{0}^{2}\right)}\right. \\
\left.+\frac{\partial}{\partial \lambda}\left[L_{m-1}^{(l)}\left(\frac{R_{k}^{2}}{R_{0}^{2}}\right)\right]\right] \cdot\left[\frac{\partial}{\partial \lambda}\left[L_{m-1}^{(l+1)}\left(\frac{R_{k}^{2}}{R_{0}^{2}}\right)\right]\right. \\
\left.-\frac{L_{m-1}^{(l+1)}\left(R_{k}^{2} / R_{0}^{2}\right)}{L_{m-1}^{(l)}\left(R_{k}^{2} / R_{0}^{2}\right)} \frac{\partial}{\partial \lambda}\left[L_{m-1}^{(l)}\left(\frac{R_{k}^{2}}{R_{0}^{2}}\right)\right]\right] \\
+\frac{L_{m-1}^{(l+1)}\left(R_{k}^{2} / R_{0}^{2}\right)}{L_{m-1}^{(l)}\left(R_{k}^{2} / R_{0}^{2}\right)}\left[\frac{2}{R_{0}}\left[\frac{\partial^{2} R_{0}}{\partial \lambda^{2}}-\frac{1}{R_{0}}\left(\frac{\partial R_{0}}{\partial \lambda}\right)^{2}\right]\right. \\
\left.\left.+\frac{1}{L_{m-1}^{(l)}\left(R_{k}^{2} / R_{0}^{2}\right)} \frac{\partial^{2}}{\partial \lambda^{2}}\left[L_{m-1}^{(l)}\left(\frac{R_{k}^{2}}{R_{0}^{2}}\right)\right]\right]\right\} .
\end{gathered}
$$

By applying recurrent expressions of orthogonal polynomial order and differentiation formulas [15, 16], first and second derivatives of the Laguerre polynomials will be obtained:

$$
\begin{gathered}
\frac{\partial}{\partial \lambda}\left[L_{m-1}^{(l)}\left(\frac{R^{2}}{R_{0}^{2}}\right)\right]=\frac{2 R^{2}}{R_{0}^{3}} L_{m-2}^{(l+1)}\left(\frac{R^{2}}{R_{0}^{2}}\right) \frac{\partial R_{0}}{\partial \lambda} \\
=\frac{2 R^{2}}{R_{0}^{3}}\left[L_{m-1}^{(l+1)}\left(\frac{R^{2}}{R_{0}^{2}}\right)-L_{m-1}^{(l)}\left(\frac{R^{2}}{R_{0}^{2}}\right)\right] \frac{\partial R_{0}}{\partial \lambda} ; \\
\frac{\partial^{2}}{\partial \lambda^{2}}\left[L_{m-1}^{(l)}\left(\frac{R^{2}}{R_{0}^{2}}\right)\right]=\frac{2 R^{2}}{R_{0}^{3}}\left\{L_{m-1}^{(l+1)}\left(\frac{R^{2}}{R_{0}^{2}}\right) \frac{\partial^{2} R_{0}}{\partial \lambda^{2}}\right. \\
\left.-L_{m-1}^{(l)}\left(\frac{R^{2}}{R_{0}^{2}}\right) \frac{\partial^{2} R_{0}}{\partial \lambda^{2}}+\frac{\partial R_{0}}{\partial \lambda} \frac{\partial}{\partial \lambda}\left[L_{m-1}^{(l+1)}\left(\frac{R^{2}}{R_{0}^{2}}\right)\right]\right\} \\
-\frac{1}{R_{0}}\left(3+\frac{2 R^{2}}{R_{0}^{2}}\right) \frac{\partial R_{0}}{\partial \lambda} \frac{\partial}{\partial \lambda}\left[L_{m-1}^{(l)}\left(\frac{R^{2}}{R_{0}^{2}}\right)\right] .
\end{gathered}
$$

Finally, by differentiating the radial mode field distribution, first- and second-order derivatives of $F_{m}^{(l)}$ are defined by the following expressions:

$$
\begin{aligned}
& \frac{\partial F_{m}^{(l)}}{\partial \lambda}=\frac{1}{R_{0}} \frac{\partial R_{0}}{\partial \lambda}\left[\frac{2 R}{R_{0}} F_{m}^{(l+1)}-F_{m}^{(l)}\left(l+\frac{R^{2}}{R_{0}^{2}}\right)\right] \\
& \frac{\partial^{2} F_{m}^{(l)}}{\partial \lambda^{2}}=\frac{2 R}{R_{0}^{2}}\left[\frac{\partial F_{m}^{(l+1)}}{\partial \lambda} \frac{\partial R_{0}}{\partial \lambda}-\frac{2}{R_{0}} F_{m}^{(l+1)}\left(\frac{\partial R_{0}}{\partial \lambda}\right)^{2}\right.
\end{aligned}
$$

$$
\begin{aligned}
& \left.+F_{m}^{(l+1)} \frac{\partial^{2} R_{0}}{\partial \lambda^{2}}+\frac{R}{R_{0}^{2}} F_{m}^{(l)}\left(\frac{\partial R_{0}}{\partial \lambda}\right)^{2}\right]+\frac{1}{R_{0}}\left(l+\frac{R^{2}}{R_{0}^{2}}\right) \\
& \cdot\left[F_{m}^{(l)} \frac{\partial^{2} R_{0}}{\partial \lambda^{2}}\right. \\
& \left.+\frac{\partial F_{m}^{(l)}}{\partial \lambda} \frac{\partial R_{0}}{\partial \lambda}-\frac{1}{R_{0}} F_{m}^{(l)}\left(\frac{\partial R_{0}}{\partial \lambda}\right)^{2}\right] .
\end{aligned}
$$

Polarization correction component $I_{2}$ first- and secondorder derivatives can be expressed analogously to $I_{1}$ in the form of finite sums:

$$
\begin{gathered}
I_{2}=\frac{(m-1) !}{(l+m-1) !} \sum_{k=0}^{N-1} G_{k}^{(2)} ; \\
\frac{\partial I_{2}}{\partial \lambda}=\frac{(m-1) !}{(l+m-1) !} \sum_{k=0}^{N-1} \frac{\partial G_{k}^{(2)}}{\partial \lambda} ; \\
\frac{\partial^{2} I_{2}}{\partial \lambda^{2}}=\frac{(m-1) !}{(l+m-1) !} \sum_{k=0}^{N-1} \frac{\partial^{2} G_{k}^{(2)}}{\partial \lambda^{2}},
\end{gathered}
$$

where

$$
\begin{aligned}
& G_{k}^{(2)}=P\left(h_{k+1}-h_{k}\right)\left[F_{m-1}^{(l)}\right]^{2} ; \\
& \frac{\partial G_{k}^{(2)}}{\partial \lambda}=P\left[F_{m}^{(l)}\right]^{2}\left(\frac{\partial h_{k+1}}{\partial \lambda}-\frac{\partial h_{k}}{\partial \lambda}\right)+G_{k}^{(2)}\left(\frac{1}{P} \frac{\partial P}{\partial \lambda}\right. \\
& \left.+\frac{2}{F_{m}^{(l)}} \frac{\partial F_{m}^{(l)}}{\partial \lambda}\right) ; \\
& \frac{\partial^{2} G_{k}^{(2)}}{\partial \lambda^{2}}=P\left[F_{m}^{(l)}\right]^{2}\left[\left(\frac{\partial h_{k+1}}{\partial \lambda}-\frac{\partial h_{k}}{\partial \lambda}\right)\right. \\
& \left.\cdot\left(\frac{1}{P} \frac{\partial P}{\partial \lambda}+\frac{2}{F_{m}^{(l)}} \frac{\partial F_{m}^{(l)}}{\partial \lambda}\right)+\frac{\partial^{2} h_{k+1}}{\partial \lambda^{2}}-\frac{\partial^{2} h_{k}}{\partial \lambda^{2}}\right] \\
& +\frac{\partial G_{k}^{(2)}}{\partial \lambda}\left(\frac{1}{P} \frac{\partial P}{\partial \lambda}+\frac{2}{F_{m}^{(l)}} \frac{\partial F_{m}^{(l)}}{\partial \lambda}\right)^{2} \\
& \quad+G_{k}^{(2)}\left\{\frac{1}{P}\left[\frac{\partial^{2} P}{\partial \lambda^{2}}-\frac{1}{P}\left(\frac{\partial P}{\partial \lambda}\right)^{2}\right]\right. \\
& \left.\quad+\frac{2}{F_{m}^{(l)}}\left[\frac{\partial^{2} F_{m}^{(l)}}{\partial \lambda^{2}}-\frac{1}{F_{m}^{(l)}}\left(\frac{\partial F_{m}^{(l)}}{\partial \lambda}\right)^{2}\right]\right\} .
\end{aligned}
$$

By differentiating characteristic equation (14) the derivatives of the normalized mode field radius will be obtained: 


$$
\frac{\partial R_{0}}{\partial \lambda}=\frac{S_{0}\left(\partial V^{2} / \partial \lambda\right)+\sum_{k=0}^{N-1}\left[\left(S_{0}-S_{1}\right)\left(h_{k}\left(\partial V^{2} / \partial \lambda\right)+V^{2}\left(\partial h_{k} / \partial \lambda\right)\right)\right]}{-2 V^{2} \cdot\left[S_{0}^{(1)}+\sum_{k=0}^{N-1} h_{k}\left(S_{1}^{(1)}-S_{2}^{(1)}\right)\right]}
$$

where

$$
\begin{aligned}
& S_{0}^{(1)}=\exp \left(-\frac{1}{R_{0}^{2}}\right) \sum_{q=0}^{2 m-2} D_{q} \sum_{p=0}^{l+q} \frac{(l+q) !}{p ! R_{0}^{2 p+3}}\left[\left(1-p R_{0}^{2}\right)^{2}\right. \\
& \left.-p R_{0}^{4}\right] ; \\
& S_{1}^{(1)}=\exp \left(-\frac{k^{2}}{N^{2} R_{0}^{2}}\right) \\
& \cdot \sum_{q=0}^{2 m-2} D_{q} \sum_{p=0}^{l+q} \frac{(l+q) ! k^{2 p}}{p ! N^{2 p} R_{0}^{2 p+3}}\left[\left(\frac{k^{2}}{N^{2}}-p R_{0}^{2}\right)^{2}-p R_{0}^{4}\right] ; \\
& S_{2}^{(1)}=\exp \left(-\frac{(k+1)^{2}}{N^{2} R_{0}^{2}}\right) \\
& \cdot \sum_{q=0}^{2 m-2} D_{q} \sum_{p=0}^{l+q} \frac{(l+q) !(k+1)^{2 p}}{p ! N^{2 p} R_{0}^{2 p+3}}\left[\left(\frac{(k+1)^{2}}{N^{2}}-p R_{0}^{2}\right)^{2}\right. \\
& \left.-p R_{0}^{4}\right] \cdot \\
& \frac{\partial^{2} R_{0}}{\partial \lambda^{2}}=-\left[V^{2} S_{0}^{(1)}+\sum_{k=0}^{N-1} h_{k}\left(S_{1}^{(1)}-S_{2}^{(1)}\right)\right]^{-1}\left\{2 \frac{\partial R_{0}}{\partial \lambda}\right. \\
& \quad \cdot \sum_{k=0}^{N-1}\left(S_{1}^{(1)}-S_{2}^{(1)}\right)\left(V^{2} \frac{\partial h_{k}}{\partial \lambda}+h_{k} \frac{\partial V^{2}}{\partial \lambda}\right)+\sum_{k=0}^{N-1}\left(S_{1}-S_{2}\right) \\
& \cdot\left(\frac{h_{k}}{2} \frac{\partial^{2} V^{2}}{\partial \lambda^{2}}+\frac{\partial h_{k}}{\partial \lambda} \frac{\partial V^{2}}{\partial \lambda}+\frac{V^{2}}{2} \frac{\partial^{2} h_{k}}{\partial \lambda^{2}}\right)+2 V^{2}\left(\frac{\partial R_{0}}{\partial \lambda}\right)^{2} \\
& \left.\cdot\left[S_{0}^{(2)}+\sum_{k=0}^{N-1} h_{k}\left(S_{1}^{(2)}-S_{2}^{(2)}\right)\right]+\frac{S_{0}}{2} \frac{\partial^{2} V^{2}}{\partial \lambda^{2}}+2 S_{0}^{(1)} \frac{\partial R_{0}}{\partial \lambda}\right\}, \\
& \left.\quad V^{2}\right\}
\end{aligned}
$$

Finally the derivatives of profile parameter $h_{k}$ defined by formula (11b) are determined by the following expressions:

$$
\begin{gathered}
\frac{\partial h_{k}}{\partial \lambda}=\frac{1}{n_{\max }^{2}-n_{N}^{2}}\left[\left(1-h_{k}\right) \frac{\partial n_{\max }^{2}}{\partial \lambda}-\frac{\partial n_{k}^{2}}{\partial \lambda}\right. \\
\left.+h_{k} \frac{\partial n_{N}^{2}}{\partial \lambda}\right] ; \\
\frac{\partial^{2} h_{k}}{\partial \lambda^{2}}=\frac{1}{n_{\max }^{2}-n_{N}^{2}}\left[\left(1-h_{k}\right) \frac{\partial^{2} n_{\max }^{2}}{\partial \lambda^{2}}-\frac{\partial^{2} n_{k}^{2}}{\partial \lambda^{2}}\right. \\
\left.+h_{k} \frac{\partial^{2} n_{N}^{2}}{\partial \lambda^{2}}-2 \frac{\partial h_{k}}{\partial \lambda}\left(\frac{\partial n_{\max }^{2}}{\partial \lambda}-\frac{\partial n_{N}^{2}}{\partial \lambda}\right)\right] .
\end{gathered}
$$

\section{Conflict of Interests}

The author declares that there is no conflict of interests regarding the publication of this paper.

where

$$
\begin{aligned}
S_{0}^{(2)} & =\exp \left(-\frac{1}{R_{0}^{2}}\right) \sum_{q=0}^{2 m-2} D_{q} \sum_{p=0}^{l+q} \frac{(l+q) !}{p ! R_{0}^{2 p+6}}\left\{\left(1-p R_{0}^{2}\right)^{3}\right. \\
& \left.+\frac{R_{0}^{2}}{2}\left[p R_{0}^{4}(3 p-1)-3\right]\right\} ; \\
S_{1}^{(2)} & =\exp \left(-\frac{k^{2}}{N^{2} R_{0}^{2}}\right)
\end{aligned}
$$

\section{References}

[1] A. Snyder and J. Love, Optical Waveguide Theory, Chapman \& Hall, London, UK, 1983.

[2] H.-G. Unger, Planar Optical Waveguides and Fibres, Clarendon Press, Oxford, UK, 1977.

[3] M. J. Adams, An Introduction to Optical Waveguides, John Wiley \& Sons, New York, NY, USA, 1981.

[4] K. S. Chiang, "Review of numerical and approximate methods for the modal analysis of general optical dielectric waveguides," Optical and Quantum Electronics, vol. 26, no. 3, pp. S113-S134, 1994. 
[5] D. Marcuse, Light Transmission Optics, Van Nostrand Reinhold, London, UK, 1972.

[6] K. Okamoto, Fundamentals of Optical Waveguides, Academic Press, San Diego, Calif, USA, 2000.

[7] A. V. Bourdine and K. A. Yablochkin, "Investigations of refractive index profile defects of silica graded-index multimode fibers of telecommunication cables," Infocommunication Technologies, vol. 8, no. 2, pp. 22-27, 2010.

[8] H. Meher and S. I. Hosain, "Variational approximations for single-mode graded-index fibers: some interesting applications," Journal of Optical Communications, vol. 24, no. 1, pp. 2530, 2003.

[9] M.-S. Wu, M.-H. Lee, and W.-H. Tsai, "Variational analysis of single-mode graded-core W-fibers," Journal of Lightwave Technology, vol. 14, no. 1, pp. 121-125, 1996.

[10] M. J. Holmes, D. M. Spirit, and F. P. Payne, "New Gaussianbased approximation for modelling non-linear effects in optical fibers," IEEE Journal of Lightwave Technology, vol. 12, no. 2, pp. 193-201, 1994.

[11] A. Ankiewicz and G.-D. Peng, "Generalized Gaussian approximation for single-mode fibers," Journal of Lightwave Technology, vol. 10, no. 1, pp. 22-27, 1992.

[12] M. I. Oksanen and I. V. Lindell, "Variational analysis of anisotropic graded-index optical fibers," IEEE Journal of Lightwave Technology, vol. 7, no. 1, pp. 87-91, 1989.

[13] R. Tewari, S. I. Hosain, and K. Thyagarajan, "Scalar variational analysis of single mode fibers with Gaussian and smoothedout profiles," Optics Communications, vol. 48, no. 3, pp. 176-180, 1983.

[14] A. Sharma, S. I. Hosain, and A. K. Ghatak, "The fundamental mode of graded-index fibres: simple and accurate variational methods," Optical and Quantum Electronics, vol. 14, no. 1, pp. $7-15,1982$.

[15] M. C. Kundu, S. Gangopadhyay, T. Basu, and S. N. Sarkar, "Evaluation of dimensionless vector and scalar propagation constants for single-mode graded index fibers by a simple technique," Optik, vol. 116, no. 11, pp. 511-514, 2005.

[16] I. Gradstein and I. Ryjik, Tables of Integrals, GIFML, Moscow, Russia, 1963.

[17] A. V. Bourdine and O. P. Delmukhametov, "Calculation of transmission parameters of the launched higher-order modes based on the combination of a modified Gaussian approximation and a finite element method," Telecommunications and Radio Engineering, vol. 72, no. 2, pp. 111-123, 2013.

[18] T. Begou, B. Bêche, N. Grossard et al., "Marcatili's extended approach: comparison to semi-vectorial methods applied to pedestal waveguide design," Journal of Optics A: Pure and Applied Optics, vol. 10, no. 5, Article ID 055310, 2008. 


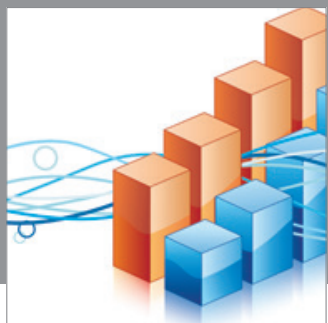

Advances in

Operations Research

mansans

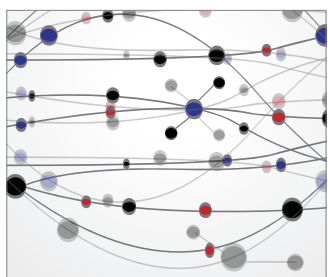

The Scientific World Journal
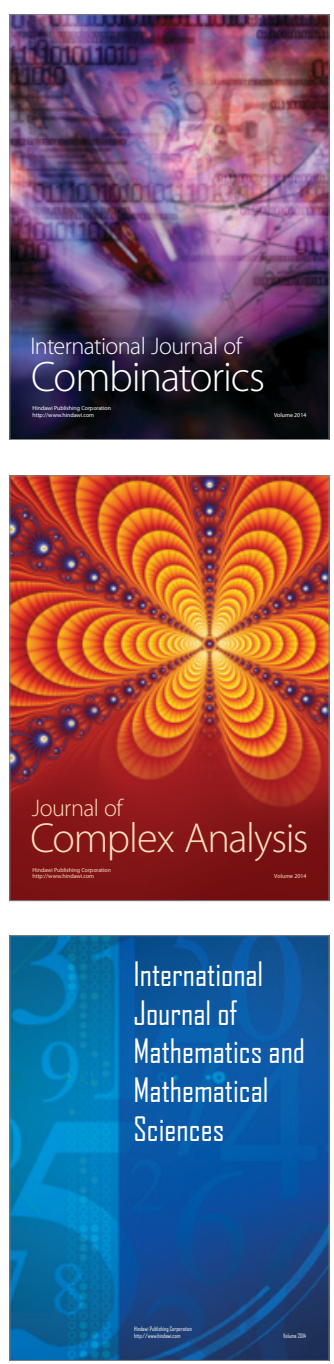
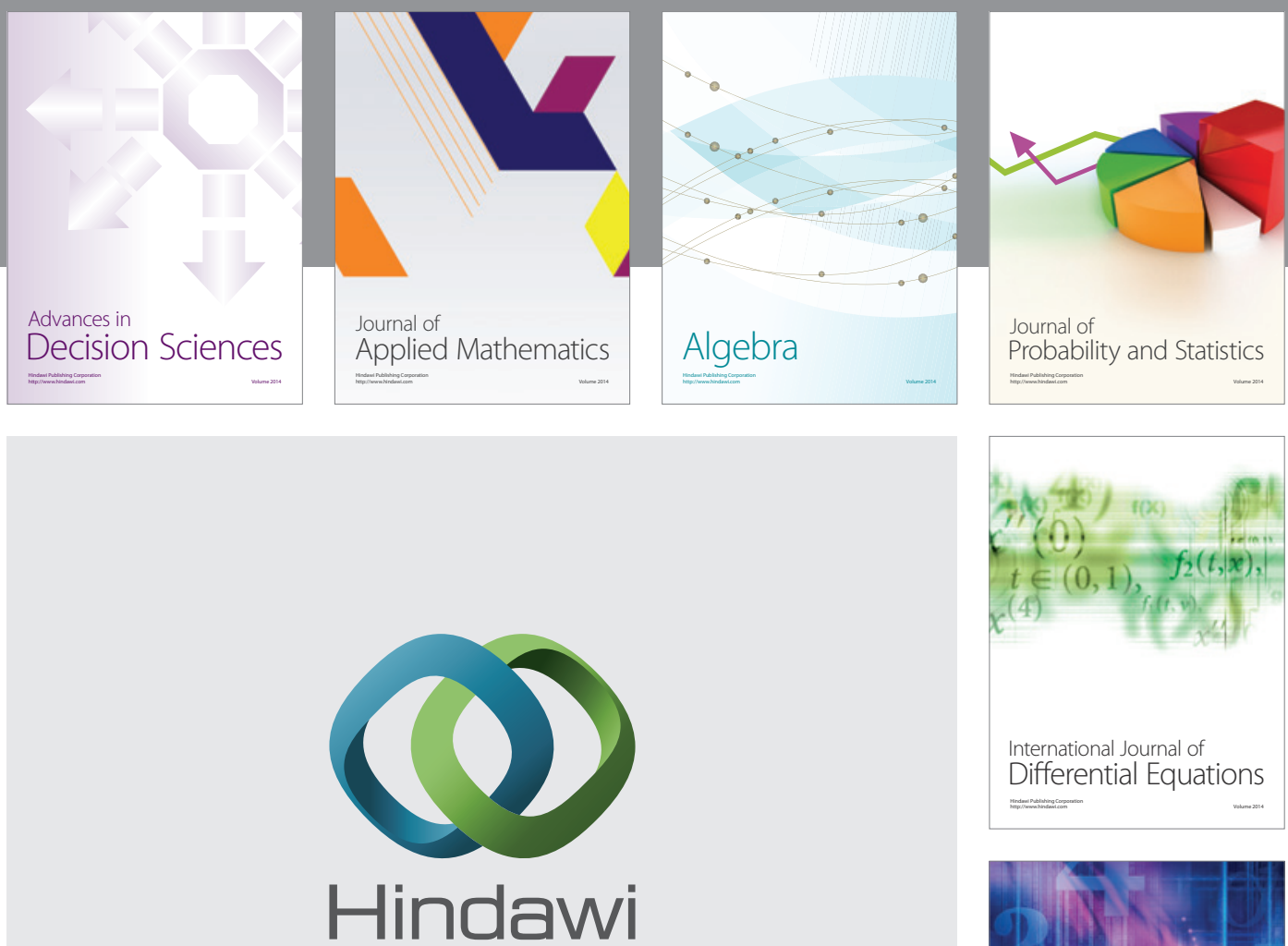

Submit your manuscripts at http://www.hindawi.com
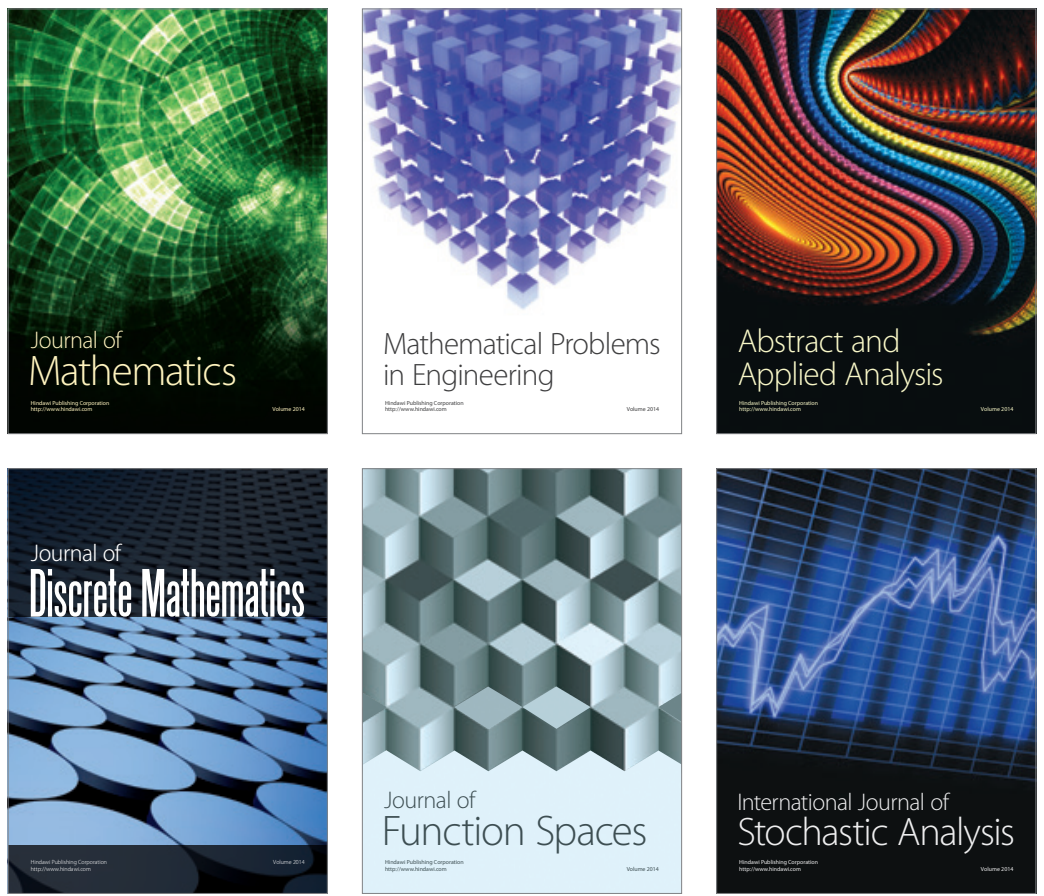

Journal of

Function Spaces

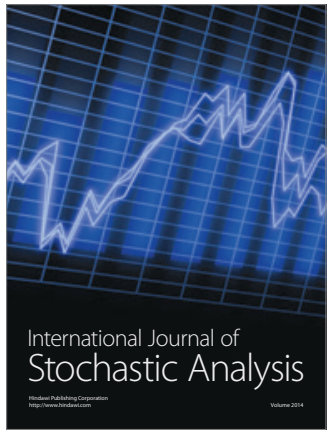

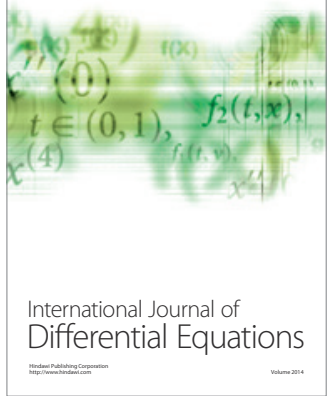
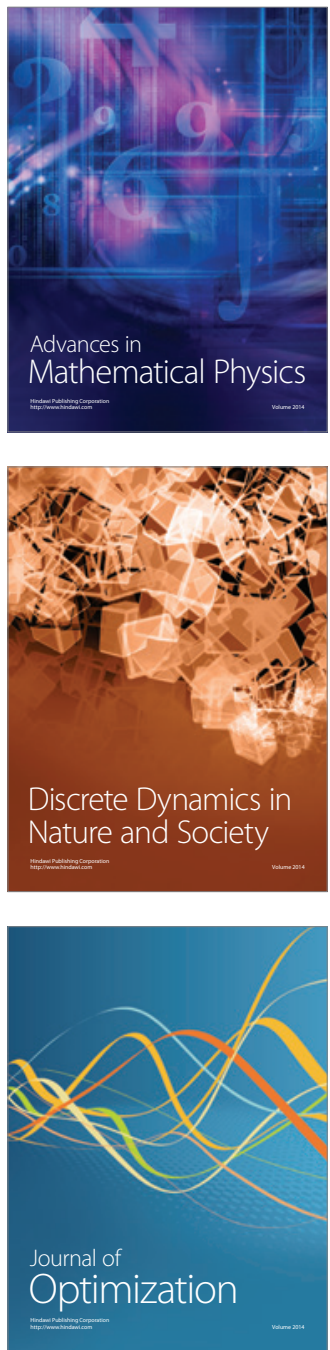